\begin{tabular}{|c|c|}
\hline Title & A modified immune network algorithm for multimodal electromagnetic problems \\
\hline Author(s) & Campelo, Felipe; Guimarães, Frederico G.; Igarashi, Hajime; Ramírez, Jaime A .; Noguchi, So \\
\hline Citation & $\begin{array}{l}\text { IEEE transactions on magnetics, 42(4), 1111-11114 } \\
\text { https://doi.org/10.1109// MA G.2006.871633 }\end{array}$ \\
\hline Issue Date & $2006-04$ \\
\hline Doc URL & http://hdl.handle.net/2115/8519 \\
\hline Rights & $\begin{array}{l}\text { (c) } 2006 \text { IEEE. Personal use of this material is permitted. However, permission to reprint/republish this material for } \\
\text { advertising or promotional purposes or for creating new collective works for resale or redistribution to servers or lists, } \\
\text { or to reuse any copyrighted component of this work in other works must be obtained from the IEEE }\end{array}$ \\
\hline Type & article \\
\hline File Information & 01608405.pdf \\
\hline
\end{tabular}

Instructions for use 


\title{
A Modified Immune Network Algorithm for Multimodal Electromagnetic Problems
}

\author{
Felipe Campelo $^{1}$, Frederico G. Guimarães ${ }^{2}$, Hajime Igarashi ${ }^{1}$, Jaime A. Ramírez ${ }^{2}$, and So Noguchi ${ }^{1}$ \\ ${ }^{1}$ Laboratory of Hybrid Systems, Graduate School of Information \\ Science and Technology, Hokkaido University, Sapporo 060-0814, Japan \\ ${ }^{2}$ Department of Electrical Engineering, Federal University of Minas Gerais, Belo Horizonte MG 31270-010, Brazil
}

\begin{abstract}
Some optimization algorithms based on theories from immunology have the feature of finding an arbitrary number of optima, including the global solution. However, this advantage comes at the cost of a large number of objective function evaluations, in most cases, prohibitive in electromagnetic design. This paper proposes a modified version of the artificial immune network algorithm (opt-AINet) for electromagnetic design optimization. The objective of this modified AINet (m-AINet) is to reduce the computational effort required by the algorithm, while keeping or improving the convergence characteristics. Another improvement proposed is to make it more suitable for constrained problems through the utilization of a specific constraint-handling technique. The results obtained over an analytical problem and the design of an electromagnetic device show the applicability of the proposed algorithm.
\end{abstract}

Index Terms-Artificial immune systems, electromagnetic design optimization, immune networks.

\section{INTRODUCTION}

$\mathbf{O}$ PTIMIZATION problems in electromagnetics are usually multivariate, nonlinear, computationally expensive, and multimodal. Frequently, not only the global optimum but also other local optima may be interesting to obtain, as it enables the designer to choose the most suitable solution from a set of optimal points.

Due to these characteristics, population-based optimization algorithms are often used for solving such problems. Many techniques exist for making stochastic algorithms find and maintain a set of suboptima [1]-[3]. The efficiency of these techniques, however, is limited by the fact that the population size is fixed, i.e., the algorithm is able to find a maximum number of optima, defined by the initial choice of parameters. Arbitrarily increasing the size of the population is seldom a good alternative, since it implies an often worthless increase in the computational cost.

The artificial immune network (AINet) is an algorithm based on the artificial immune systems (AIS) paradigm [4]. This algorithm was inspired by the idiotypic network theory for explaining the immune system dynamics, originally proposed in [5]. The optimization version of the AINet [6], [7] is called opt-AINet, and presents a number of interesting features, such as dynamic variation of the population size, local and global search, and the ability to maintain any number of optima. These are highly desirable characteristics, but they are obtained at the cost of a very large number of objective function evaluations. In the context of numerical electromagnetic problems, this price may be prohibitive.

In this paper, we present a modified version of the opt-AINet, which requires a lower number of objective function evaluations without losing the valuable characteristics of the original algorithm. Additionally, a specific constraint-handling technique has been introduced. A comparison between the characteristics and performance of the opt-AINet and the modified ver-

Digital Object Identifier 10.1109/TMAG.2006.871633 sion proposed here (m-AINet) is presented, as well as results for showing the applicability of the proposed algorithm for electromagnetic optimization.

\section{Previous WORK}

The AINet algorithm was originally proposed for performing data analysis tasks [8]. It was later used for multimodal optimization (opt-AINet) [6]. This algorithm is capable of performing local and global search, as well as to dinamically adjust the size of the population. The local search is based in the operations of cloning, maturation, and selection of the antibodies (points in the search space), while the global exploration is done by the insertion of random points and variation in the size of the population.

The cloning operator basically gives each point a user-defined number of exact copies, or clones. An antibody and its clones are called a subpopulation. The maturation operator submits each clone to Gaussian noise, with the standard deviation as a function of the normalized fitness of the original antibody. The fitness of the maturated clones is evaluated and only the best point from each subpopulation is allowed to proceed in the optimization process, while the others are excluded.

The above cycle is repeated until the average fitness of the population does not present a significant variation between two iterations. When this is achieved, the population is said to have reached global stabilization, and the algorithm proceeds to the suppression operator: the similarity (Euclidean distance) is determined among all the antibodies. If two points are closer than a user-defined threshold $\sigma$, the one with lower fitness is eliminated, in order to avoid redundancy. The surviving antibodies, after the suppression operation, are called memory antibodies.

After the suppression, the size of the population is increased by a fraction $d \%$ of the number of surviving antibodies. The new antibodies are randomly generated, and the cloning/maturation/selection cycle is restarted. The algorithm continues until the number of memory antibodies does not vary between two successive suppressions, or the algorithm performs an user-de- 
fined maximum number of iterations. For a more detailed description of the opt-AINet algorithm, see [6].

Comparative tests between opt-AINet and other algorithms have been performed in [7]. The reported results show that, while the algorithm was successful in finding the global optimum of the test functions used, the number of function evaluations needed was much bigger than that for the other algorithms used for comparison. While the paper concludes that this heavy computational cost can be somehow related to the increase in the size of the population, it does not explore the possibility of the populational growth being caused by the finding of local optima. However, even if this is the case, such a large number of function evaluations makes the algorithm unsuitable for electromagnetic optimization.

Another limitation of the opt-AINet is the lack of a mechanism for dealing with constraints. Although it is possible to use the penalization of the objective function by the constraint violations to transform a constrained problem into an unconstrained one, this could lead the opt-AINet algorithm to converge to a large number of unfeasible optima, thus increasing even more the computational cost. This is especially true in heavily constrained spaces or when dealing with equality constraints.

\section{The Modified Ainet AlgorithM}

In order to reduce the computational cost of the opt-AINet, as well as enabling the solution of constrained problems, a modified algorithm has been developed. The m-AINet algorithm shares the overall structure of the opt-AINet, but most of its operators have been altered, as described in this session.

Consider a general mono-objective optimization problem

$$
\begin{aligned}
& \max f(\mathbf{x}) \in \mathbb{R} \\
& \text { subject to }: \begin{cases}g_{i}(\mathbf{x}) \leq 0, & i=1, \ldots, p \\
h_{j}(\mathbf{x})=0, & j=1, \ldots, q\end{cases}
\end{aligned}
$$

where $f(\cdot): \mathbb{R}^{n} \mapsto \mathbb{R}$ is the objective function, $g_{i}(\cdot): \mathbb{R}^{n} \mapsto$ $\mathbb{R}$ are the inequality constraints and $h_{j}(\cdot): \mathbb{R}^{n} \mapsto \mathbb{R}$ are the equality constraints. As in the opt-AINet, the m-AINet starts by generating an initial population of npop $_{0}$ antibodies in the normalized search space, and evaluating these points over the objective and constraint functions. The fitness of a given antibody is equal to the value of the objective function for the point, in case of maximization problems, or the negative of this value, for minimization.

Each point then receives two clones for each dimension of the problem. These clones are symmetrically displaced from the original antibody by a distance $k$

$$
k=0.1 \exp \left(-f^{*}\right)(1+\operatorname{Gauss}(0,1))
$$

where $f^{*}$ is the normalized fitness of the original antibody, and Gauss $(0,1)$ is a random Gaussian variable. Thus, after this step, each antibody will have $2 n$ clones equally displaced over each dimension.

The clones are evaluated over the objective function and the best point from each dimension is used for estimating the best vertex of the hypercube defined by the $2 n$ clones. An extra clone is generated at the estimated best vertex, as shown in Fig. 1. This mutation scheme has been inspired by a technique

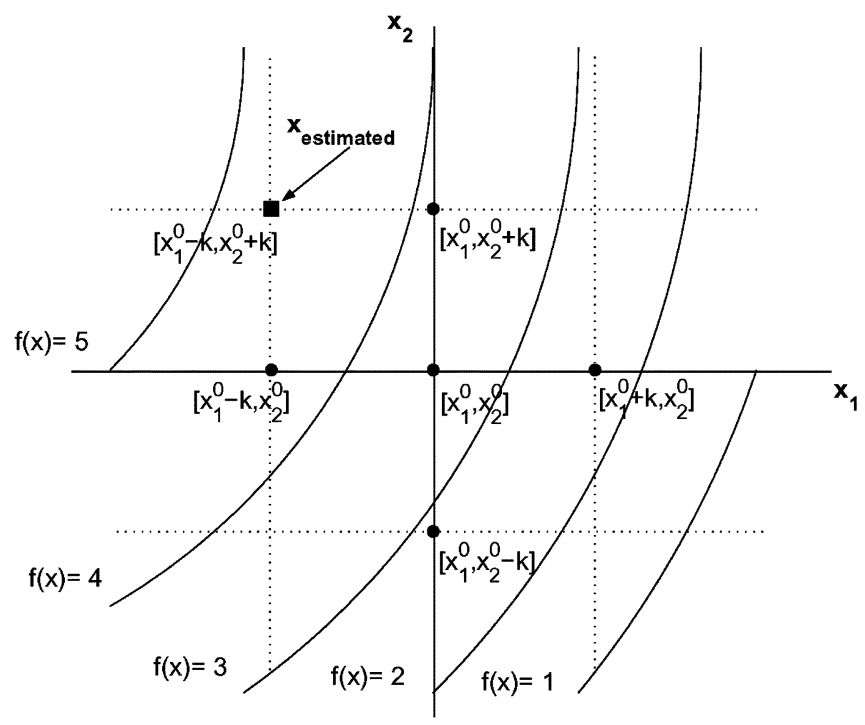

Fig. 1. Hypercubic maturation and best vertex estimation for an unconstrained two-dimensional maximization problem.

called worst vertex prediction, originally used in robust optimization algorithms [9]. It is hereafter called hypercubic maturation, in contrast with the random Gaussian maturation from the opt-AINet. In the m-AINet, the number of clones for each subpopulation is no longer a free parameter, but instead a fixed number $2 n+1$. This is an interesting feature, since the performance of the opt-AINet algorithm is very sensitive to variations in the number of clones. An antibody and its maturated clones form a subpopulation of points.

Only the best point from each subpopulation is selected for surviving. The m-AINet uses a feasibility-based selection criterion [10], which takes into account the constraint violations. The selection is done according to the following rules.

- Between two feasible solutions, the one with the highest fitness value is selected.

- If one solution is feasible and the other is unfeasible, the feasible is selected; however, in a small percentage of the cases, the fitness value of the violating point is used and the points are compared as in the item above. This mechanism helps maintaining the diversity in the population.

- If both solutions are unfeasible, the one with the lowest sum of constraint violations is selected.

These selection rules ensure a strong convergence pressure toward the feasible space, without the need of determining any penalization constant. Moreover, when combined with the hypercubic maturation, it also avoids the detection of unfeasible peaks by driving the subpopulations toward the feasible space. It is important to notice here that, in the case of constrained problems, this scheme is also used for the estimation of the best vertex.

Each subpopulation is then checked for stabilization: if the surviving point did not change since the last iteration, the subpopulation is said to have stabilized and is removed from the iterative cycle. This criterion is called partial stabilization, in contrast with the global stabilization criterion used in the optAINet. By avoiding the evaluation of already stabilized subpopulations, this scheme greatly reduces computational cost of the algorithm. It is especially effective when combined with the 
hypercubic maturation, since in this case there is a certain degree of determinism in the local search, even though it is not a fully deterministic search, as it uses a Gaussian variation of the hypercube size. The iterative cycle continues until all the subpopulations have stabilized or a maximum number of iterations (maxgen) is achieved. The algorithm then proceeds for the suppression operator.

The suppression is performed like in the opt-AINet, but again using the feasibility-based selection in case of close points. Moreover, only the points that did not stabilize during the iterative cycle return to the active population, while the others are stored in an external memory population. This memory population is only mixed with the active population for the consecutive suppressions: no more function evaluations are required for the memory antibodies.

Finally, a number of new randomly generated antibodies are added to the nonstabilized antibodies and this new active population returns to the iterative cycle. The number of new antibodies is a fraction $d \%$ of the memory population size. This process is repeated until the size of the memory population does not vary for two consecutive generations, or if the algorithm reaches a user-defined maximum number of suppressions (maxsup).

The code for the m-AINet as it is described here has been implemented in Matlab, and is available at [11].

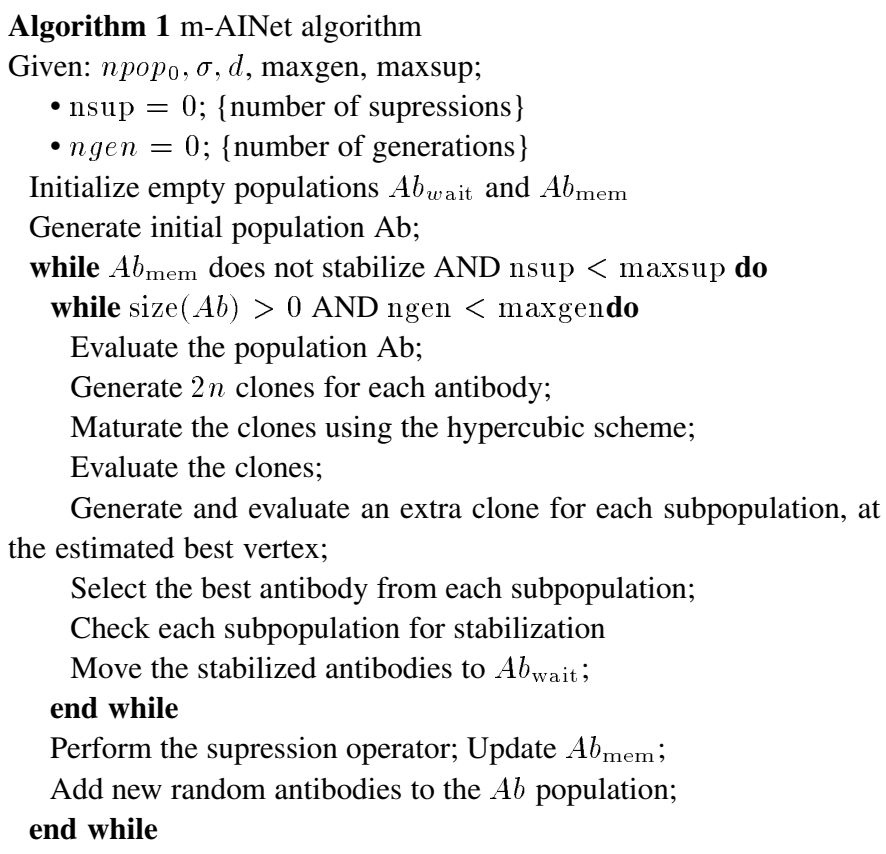

\section{RESULTS}

\section{A. Analytic Constrained Problem}

For testing the ability of the m-AINet to work on constrained search spaces, the following minimization problem was considered:

$$
\begin{aligned}
\min f(\mathbf{x})= & \alpha n+\sum_{i=1}^{n} x_{i}^{2}-\alpha \cos \left(2 \pi x_{i}\right) \\
\text { subject to }: & \begin{array}{l}
g(\mathbf{x})=-x_{2}-1.5 \leq 0, \\
h(\mathbf{x})=x_{1}-1=0
\end{array}
\end{aligned}
$$

TABLE I

RESULTS FOR THE CONSTRAINED PROBLEM

\begin{tabular}{l|cccc}
\hline & \%Conv & Optima(Total) & Optima(Feasible) & Evals \\
\hline m-AINet & $86 \%$ & $49.4 \pm 3.37$ & $28.5 \pm 3.62$ & $8873 \pm 639$ \\
opt-AINet & $80 \%$ & $250 \pm 6.09$ & $6.8 \pm 2.86$ & 341690 \\
& & & & \pm 12900 \\
\hline
\end{tabular}

with $\alpha=10, n=3$, and $\left[-5.12 \leq x_{i} \leq 5.12\right]$. The objective function is the three-dimensional (3-D) Rastrigin function. Its unconstrained version presents $10^{n}$ peaks over the search space, but the constraints, in particular the equality one, greatly reduces the number of feasible peaks. The constrained global optimum is located at the point $[1,0,0]$, and the convergence criterion used was $\left\|x_{\text {best }}-[1,0,0]\right\|_{2} \leq \sqrt{0.02}$, as suggested in [12]. The tolerance for the equality constraint was set to $h t o l=0.05$, which means that points with values for this constraint lower than $h t o l$ are considered feasible.

The results obtained by the m-AINet are shown in Table I. These results were obtained by using the following parameters: npop $_{0}=20, \sigma=0.05, d=0.5$, maxgen $=30$, and $\operatorname{maxsup}=10$.

For analyzing the effects of the proposed modifications on the performance of the algorithm, we have also tested the opt-AINet over this problem. The parameters used were the same as used for the m-AINet, with the additinal parameters $N_{C}=7$ and $\beta=$ 100 . The number of clones was intentionally chosen as $2 n+1$, i.e., the number of clones generated for each subpopulation was the same for the opt-AINet and the m-AINet. The opt-AINet used the penalization of the objective function as the method for dealing with the constraints. The penalty function constant used was $K_{p}=10$.

Table I shows the effect of the proposed modifications on the performance and computational cost of the m-AINet when compared to the original opt-AINet. The convergence rate improved slighly, but the biggest improvement was the reduction of the computational cost, represented by the number of objective function evaluations (Evals column). This can be justified by the use of the partial stabilization of the subpopulations. This strategy is particularly successful when combined with the hypercubic maturation: in the random Gaussian maturation the chance of a subpopulation to improve after stopping for one generation is still relatively big. On the other hand, due to a certain degree of determinism introduced by the hypercubic maturation, this possibility is much smaller. Thus, the hypercubic maturation allows the use of the partial stabilizations technique without interfeering on the evolution of the subpopulations.

Additionally, the use of the feasibility-based selection criterion seems to have enabled the m-AINet to concentrate on the search for the feasible peaks, ignoring almost all the unfeasible ones (the number of unfeasible peaks is given by the difference between Optima(Total) and Optima(Feasible)). This is a valuable characteristic that contributed not only for the improvement in convergence and in the number of feasible peaks found, but also for the reduction on the computational cost, since the size of the population is a function of the amount of peaks found.

\section{B. Electromagnetic Problem}

The proposed algorithm was also tested on the design of an electromagnetic device. The TEAM Benchmark Problem 22 [13] consists on the minimization of the magnetic flux 
TABLE II

RESULTS FOR THE SMES PROBLEM

\begin{tabular}{l|cccc}
\hline & m-AINet(1) & m-AINet(2) & GA [12] & Best [13] \\
\hline$r_{2}[m]$ & 2.99 & 3.26 & 3.05 & 3.08 \\
$h_{2}[m]$ & 0.524 & 0.872 & 0.492 & 0.478 \\
$d_{2}[m]$ & 0.381 & 0.190 & 0.400 & 0.394 \\
$B_{\text {Stray }[m T]}$ & 0.8039 & 1.0269 & 0.9360 & 0.8896 \\
Energy $[M J]$ & 173.93 & 181.17 & 179.74 & 180.03 \\
\hline
\end{tabular}

density at a certain distance from a superconducting magnetic energy storage (SMES) device. In this paper, we have used the 3 -D version of this problem. The design parameters are three geometric variables which define the size and position of the outer coil of the device. The limits of the search space and other specific details of this problem are discussed in [13].

As stated above, the problem is defined as the minimization of the value of $\mathbf{B}_{\text {Stray }}$, subject to

$$
\begin{aligned}
& B_{\max } \leq 4.92 T \\
& \frac{\text { Energy }-180 \mathrm{MJ}}{180 \mathrm{MJ}}=0
\end{aligned}
$$

where $B_{\max }[T]$ is the maximum magnetic flux density at the outer coil, and Energy [MJ] is the amount of energy stored by the device. In this paper, the tolerance for the energy constraint has been set at $5 \%$ of the reference value of $180 \mathrm{MJ}$.

The m-AINet has been applied to solve this problem, with the following configuration: npop $_{0}=10, \sigma=0.04, d=$ 1 , maxgen $=20$, and maxsup $=10$. The optimization process required 2400 evaluations of the device, and returned 10 feasible optima, at a relative cost of 240 evaluations per optimum. Two of the best points found are shown in Table II, together with other solutions available in the literature. The number of function evaluations required by the GA [12] was, by coincidence, also 2400. This value is not available for the best solution known [13].

From Table II, one can see that the solutions obtained by $\mathrm{m}$-AINet are comparable to other results available in the literature. The first result (m-AINet(1)) presents a very low value for the objective function, with a violation of the energy constraint within the $5 \%$ tolerance specified. Solution m-AINet(2), on the other hand, shows a slighly larger value for $B_{\text {Stray }}$, and a very small deviation from the reference value for Energy. The fact that the proposed algorithm was able to find multiple optima, while requiring the same number of function evaluations as a GA, is an interesting feature. These additional optima provide a range of design options for the designer, who may decide, for example, for a solution with lower sensitivity to variations in the design parameters, even if it presents a slightly higher value for the objective.

\section{CONCLUSION}

A modified version of the artificial immune network algorithm for multimodal optimization has been proposed. The main differences between the m-AINet and the original opt-AINet algorithm are the use of the partial stabilizations criterion, the inclusion of a specific scheme for dealing with constrained problems, and the use of the hypercubic maturation. With these mod- ifications, the resulting algorithm was able not only to maintain the interesting characteristics of the opt-AINet, but also to greatly reduce the number of objective function evaluations required in the optimization process. This reduction makes the algorithm suitable for electromagnetic problems. The need of a user-defined value for the number of clones has also been removed in the m-AINet.

We remark that the proposed algorithm allows the determination of multiple optimal solutions, including the global one, at an acceptable cost. Subsequently, the designer may analyze these solutions with respect to other characteristics not initially considered during the optimization process, such as sensitivity analysis or more subjective criteria, not easily described in mathematical form.

\section{ACKNOWLEDGMENT}

This work was supported by the National Council of Scientific and Technologic Development - CNPq - under Grant 141731/2004-4 and Grant 300353/1997-9, Brazil, and by the Ministry of Education, Culture, Sports, Science and Technology: MEXT, Japan.

\section{REFERENCES}

[1] D. E. Goldberg and J. J. Richardson, "Genetic algorithms with sharing for multimodal function optimization," in Proc. 2nd Int. Conf. Genetic Algorithms, Cambridge, MA, July 1987, pp. 41-49.

[2] S. W. Mahfoud, "Crowding and preselection revisited," in Proc. 2nd Conf. Parallel Problem Solving from Nature, Brussels, Belgium, Sept. 1992.

[3] F. Campelo, F. G. Guimarães, and J. A. Ramírez, "A clonal selection algorithm for optimization in electromagnetics," IEEE Trans. Magn., vol. 41, no. 5, pp. 1736-1739, May 2005.

[4] L. N. de Castro and J. Timmis, Artificial Immune Systems: A New Computational Intelligence Approach. Berlin, Germany: Springer-Verlag, 2002.

[5] N. K. Jerne, "Toward a Network Theory of the Immune System," Ann. Immunol., (Inst. Pasteur), vol. 125C, pp. 373-389, 1974.

[6] L. N. de Castro and J. Timmis, "An artificial immune network for multimodal function optimization," in Proc. IEEE Congr. Evolutionary Computation, vol. 1, May 2002, pp. 699-674.

[7] J. Timmis, C. Edmonds, and J. Kelsey, "Assessing the performance of two immune inspired algorithms and a hybrid genetic algorithm for function optimization," in Proc. IEEE Congr. Evolutionary Computation, vol. 1, Jul. 2004, pp. 1044-1051.

[8] L. N. de Castro and F. J. von Zuben, "aiNet: An Artificial Immune Network for Data Analysis," in Data Mining: A Heuristic Approach, H. Abbass, R. Sarker, and C. Newton, Eds. Sydney, Australia: Idea Group Publishing, Univ. New South Wales, 2001.

[9] P. Alotto, C. Magele, W. Renhart, G. Steiner, and A. Weber, "Robust target functions in electromagnetic design," COMPEL, vol. 22, no. 3 , pp. 549-560, 2003.

[10] E. Mezura-Montes and C. A. C. Coello, "A simple multimembered evolution strategy to solve constrained optimization problems," IEEE Trans. Evol. Comp., vol. 9, no. 1, pp. 1-17, Feb. 2005.

[11] F. Campelo. Matlab Code for the m-AINet Algorithm. [Online]. Available: http://www.fcampelofp.hpg.ig.com.br/

[12] R. H. C. Takahashi, J. A. Vasconcelos, J. A. Ramirez, and L. Krahenbuhl, "A multiobjective methodology for evaluating genetic operators," IEEE Trans. Magnetics, vol. 39, no. 3, pp. 1321-1324, May 2003.

[13] TEAM Workshop Problem 22: SMES Optimization Benchmark [Online]. Available: http://www-igte.tu-graz.ac.at/archive/ team/team3dis.htm

Manuscript received June 20, 2005 (e-mail: pinto@em-si.eng.hokudai.ac.jp). 